\title{
Antideuterons as a signature of supersymmetric dark matter
}

\author{
Fiorenza Donato* \\ Laboratoire de Physique Théorique LAPTH, BP110, F-74941 Annecy-le-Vieux Cedex, France
}

\author{
Nicolao Fornengo ${ }^{\dagger}$ \\ Instituto de Física corpuscular-C.S.I.C., Departamento de Física Teòrica, Universitat de Valencia, C./Dr Moliner 50, \\ E-46100 Burjassot, Valéncia, Spain \\ Pierre Salati \\ Laboratoire de Physique Théorique LAPTH, BP110, F-74941 Annecy-le-Vieux Cedex, France \\ and Universite de Savoie, BP1104 73011 Chambéry Cedex, France \\ (Received 28 April 1999; published 17 July 2000)
}

\begin{abstract}
Once the energy spectrum of the secondary component is well understood, measurements of the antiproton cosmic-ray flux at Earth will be a powerful way to indirectly probe for the existence of supersymmetric relics in the galactic halo. Unfortunately, it is still spoiled by considerable theoretical uncertainties. As shown in this work, searches for low-energy antideuterons appear in the meantime as a plausible alternative, worth being explored. Above a few $\mathrm{GeV} / n$, a dozen spallation antideuterons should be collected by the future Alpha Magnetic Spectrometer experiment on board International Space Station Alpha. For energies less than $\sim 3 \mathrm{GeV} / n$, the $\bar{D}$ spallation component becomes negligible and may be supplanted by a potential supersymmetric signal. If a few low-energy antideuterons are discovered, this should be seriously taken as a clue for the existence of massive neutralinos in the Milky Way.
\end{abstract}

PACS number(s): 98.70.Sa, 14.20.-c, 14.80.Ly, 95.35.+d

\section{INTRODUCTION}

Cosmic ray fluxes are about to be measured with unprecedented precision both by balloon borne detectors and by space instruments. The various ongoing experiments are also hunting for traces of antimatter in cosmic radiation. The BESS collaboration [1] plans to push the limit on the $\overline{\mathrm{He}} / \mathrm{He}$ ratio down to $10^{-8}$ whereas the Alpha Magnetic Spectrometer (AMS) should reach a sensitivity of $\sim 10^{-9}$ once it is installed on the International Space Station Alpha (ISSA) [2]. The search for antinuclei has profound cosmological implications. The discovery of a single antihelium or anticarbon would actually be a smoking gun for the existence of antimatter islands in our neighborhood. However, light antinuclei, mostly antiprotons but also antideuterons, are actually produced in our Galaxy as secondaries. They result from the interaction of high-energy cosmic-ray protons with the interstellar gas of the Milky Way disk. In a previous analysis, Chardonnet et al. [3] have estimated the flux of antideuterium $\bar{D}$ and antihelium ${ }^{3} \overline{\mathrm{H}}$ e secondaries. The $\bar{D}$ signal is very weak but may marginally be detected by AMS on board ISSA. The case of antihelium is, at least for the moment, hopeless.

The dark matter of the Milky Way could be made mostly of elementary particles such as the heavy and neutral species predicted by supersymmetry. The mutual annihilations of these relics, potentially concealed in the halo of our Galaxy,

\footnotetext{
*Email address: donato@lapp.in2p3.fr

†Email address: fornengo@flamenco.ific.uv.es

"Email address: salati@lapp.in2p3.fr
}

would therefore produce an excess in the cosmic radiation of gamma rays, antiprotons, and positrons. In particular, supersymmetric antiprotons should be abundant at low energy, a region where the flux of $\bar{p}$ secondaries is a priori negligible. There is quite a bit of excitement in trying to extract from the observations a possible $\bar{p}$ exotic component which would signal the presence of supersymmetric dark matter in the Galaxy. Unfortunately, it has been recently realized [4-6] that a few processes add up together to flatten out, at low energy, the spectrum of secondary antiprotons. Ionization losses as well as inelastic but nonannihilating scatterings on the hydrogen atoms of the galactic disk result in the decrease of the antiproton energy. The low-energy tail of the $\bar{p}$ spectrum is replenished by the more abundant population from higher energies. That effect is further strengthened by solar modulation which also shifts the energy spectrum towards lower energies. As a result of these effects, the secondary $\bar{p}$ 's are much more abundant at low energy than previously thought. Disentangling an exotic supersymmetric contribution from the conventional component of spallation antiprotons may turn out to be a very difficult task. The antiproton signal of supersymmetric dark matter is therefore in jeopardy.

Antideuterons, i.e., the nuclei of antideuterium, are free from such problems. As explained in Sec. II, they form when an antiproton and an antineutron merge. The two antinucleons must be at rest with respect to each other in order for fusion to take place successfully. For kinematic reasons, a spallation reaction creates very few low-energy particles. Low-energy secondary antideuterons are even further suppressed. Energy loss mechanisms are also less efficient in shifting the antideuteron energy spectrum towards low energies. The corresponding interstellar (IS) flux is derived in 
Sec. III, for energies in the range extending from 0.1 up to $100 \mathrm{GeV} / n$. It reaches a maximum of $(2-5) \times 10^{-8} \bar{D} \mathrm{~m}^{-2} \mathrm{~s}^{-1} \mathrm{sr}^{-1} \mathrm{GeV}^{-1}$ for a kinetic energy of $\sim 4 \mathrm{GeV} / n$. A dozen secondary antideuterons should be collected by the AMS-ISSA experiment.

On the other hand, supersymmetric $\bar{D}$ 's are manufactured at rest with respect to the Galaxy. In neutralino annihilations, antinucleons are predominantly produced with low energies. This feature is further enhanced by their subsequent fusion into antideuterons, hence a fairly flat spectrum for supersymmetric antideuterium nuclei as shown in Sec. IV. Below a few $\mathrm{GeV} / n$, secondary antideuterons are quite suppressed with respect to their supersymmetric partners. That lowenergy suppression is orders of magnitude more effective for antideuterons than for antiprotons. This makes cosmic-ray antideuterons a much better probe of supersymmetric dark matter than antiprotons.

Unfortunately, antideuteron fluxes are quite small with respect to $\bar{p}$ 's. We nevertheless show in Sec. V that a significant portion of the supersymmetric parameter space may be explored by measuring the cosmic-ray $\bar{D}$ flux at low energy. In particular, an AMS-ISSA caliber experiment should reach a sensitivity of $4.8 \times 10^{-8} \bar{D} \mathrm{~m}^{-2} \mathrm{~s}^{-1} \mathrm{sr}^{-1} \mathrm{GeV}^{-1}$ at solar minimum, pushing it down to $3.2 \times 10^{-8}$ $\times \bar{D} \mathrm{~m}^{-2} \mathrm{~s}^{-1} \mathrm{sr}^{-1} \mathrm{GeV}^{-1}$ at solar maximum, for a modulated energy of $0.24 \mathrm{GeV} / n$.

\section{PRODUCTION OF ANTIDEUTERONS}

At this point, our goal is to derive the cross section for the production of antideuterons. The processes at stake are both the spallation of a cosmic-ray high-energy proton on an hydrogen atom at rest and the annihilation of a neutralino pair. The number $d \mathcal{N}_{X}$ of particles $X$-antinucleons or antideuterons - produced in a single reaction and whose momenta are $\overrightarrow{k_{X}}$, is related to the differential production cross section through

$$
d \mathcal{N}_{X}=\frac{1}{\sigma_{\text {tot }}} d^{3} \sigma_{X}\left(\sqrt{s}, \overrightarrow{k_{X}}\right)
$$

where $\sigma_{\text {tot }}$ denotes the total cross section for the process under scrutiny - spallation reaction or neutralino annihilation. The total available energy is $\sqrt{s}$. The corresponding differential probability for the production of $X$ is defined as

$$
d \mathcal{N}_{X}=\mathcal{F}_{X}\left(\sqrt{s}, \vec{k}_{X}\right) d^{3} \vec{k}_{X}
$$

For each of the processes under concern, the differential probability for the production of an antiproton or an antineutron may be derived. The calculation of the probability for the formation of an antideuteron can now proceed in two steps. We first need to estimate the probability for the creation of an antiproton-antineutron pair. Then, those antinucleons merge to yield an antinucleus of deuterium.

As explained in Ref. [3], the production of two antinucleons is assumed to be proportional to the square of the production of one of them. The hypothesis that factorization of the probabilities holds is fairly well established at high energies. For spallation reactions, however, the bulk of the antiproton production takes place for an energy $\sqrt{s} \sim 10 \mathrm{GeV}$ which turns out to be of the same order of magnitude as the antideuteron mass. Pure factorization should break in that case as a result of energy conservation. It needs to be slightly adjusted. We have therefore assumed that the center of mass energy available for the production of the second antinucleon is reduced by twice the energy carried away by the first antinucleon

$$
\begin{aligned}
\mathcal{F}_{\bar{p}, \bar{n}}\left(\sqrt{s}, \overrightarrow{k_{\bar{p}}}, \overrightarrow{k_{\bar{n}}}\right)= & \frac{1}{2} \mathcal{F}_{\bar{p}}\left(\sqrt{s}, \overrightarrow{k_{\bar{p}}}\right) \mathcal{F}_{\bar{n}}\left(\sqrt{s}-2 E_{\bar{p}}, \overrightarrow{k_{\bar{n}}}\right) \\
& +\left(\overrightarrow{k_{\bar{p}}} \leftrightarrow \overrightarrow{k_{\bar{n}}}\right)
\end{aligned}
$$

Once the antiproton and the antineutron are formed, they combine to give an antideuteron with probability

$$
\mathcal{F}_{\bar{D}}\left(\sqrt{s}, \overrightarrow{k_{\bar{D}}}\right) d^{3} \overrightarrow{k_{\bar{D}}}=\int d^{3} \overrightarrow{k_{\bar{p}}} d^{3} \overrightarrow{k_{\bar{n}}} \mathcal{C}\left(\overrightarrow{k_{\bar{p}}}, \overrightarrow{k_{\bar{n}}}\right) \mathcal{F}_{\bar{p}, \bar{n}}\left(\sqrt{s}, \overrightarrow{k_{\bar{p}}}, \overrightarrow{k_{\bar{n}}}\right)
$$

The summation is performed on those antinucleon configurations for which

$$
\overrightarrow{k_{\bar{p}}}+\overrightarrow{k_{\bar{n}}}=\overrightarrow{k_{D}}
$$

The coalescence function $\mathcal{C}\left(\overrightarrow{k_{\bar{p}}}, \overrightarrow{k_{\bar{n}}}\right)$ describes the probability for a $\bar{p}-\bar{n}$ pair to yield by fusion an antideuteron. That function depends actually on the difference $\overrightarrow{k_{\bar{p}}}-\overrightarrow{k_{\bar{n}}}=2 \vec{\Delta}$ between the antinucleon momenta so that relation (4) may be expressed as

$$
\begin{aligned}
\mathcal{F}_{\bar{D}}\left(\sqrt{s,} \overrightarrow{k_{D}}\right)= & \int d^{3} \vec{\Delta} \mathcal{C}(\vec{\Delta}) \\
& \times \mathcal{F}_{\bar{p}, \bar{n}}\left(\sqrt{s}, \overrightarrow{k_{\bar{p}}}=\frac{\overrightarrow{k_{D}}}{2}+\vec{\Delta}, \overrightarrow{k_{\bar{n}}}=\frac{\overrightarrow{k_{\bar{D}}}}{2}-\vec{\Delta}\right) .
\end{aligned}
$$

An energy of $\sim 3.7 \mathrm{GeV}$ is required to form by spallation an antideuteron, whereas the binding energy of the latter is $B$ $\sim 2.2 \mathrm{MeV}$. The coalescence function is therefore strongly peaked around $\vec{\Delta}=\overrightarrow{0}$ and expression (6) simplifies into

$$
\mathcal{F}_{\bar{D}}\left(\sqrt{s}, \overrightarrow{k_{\bar{D}}}\right) \simeq\left\{\int d^{3} \vec{\Delta} \mathcal{C}(\vec{\Delta})\right\} \mathcal{F}_{\bar{p}, \bar{n}}\left(\sqrt{s}, \overrightarrow{k_{\bar{p}}}=\frac{\overrightarrow{k_{\bar{D}}}}{2}, \overrightarrow{k_{\bar{n}}}=\frac{\overrightarrow{k_{D}}}{2}\right),
$$

where the probability for the formation of the $\bar{p}-\bar{n}$ pair has been factored out. The term in brackets may be estimated in the rest frame of the antideuteron through the Lorentz invariant term

$$
\int \frac{E_{\bar{D}}^{-}}{E_{\bar{p}} E_{\bar{n}}} d^{3} \vec{\Delta} \mathcal{C}(\vec{\Delta}) \simeq\left(\frac{m_{\bar{D}}^{-}}{m_{\bar{p}} m_{\bar{n}}}\right)\left(\frac{4}{3} \pi P_{\text {coal }}^{3}\right)
$$


In that frame, the antinucleons merge if the momentum of the corresponding two-body reduced system is less than some critical value $P_{\text {coal }}$. That coalescence momentum is the only free parameter of our factorization and coalescence scheme. As shown in Ref. [3], the resulting antideuteron production cross section in proton-proton collisions is well fitted by this simple one-parameter model. A value of $P_{\text {coal }}$ $=58 \mathrm{MeV}$ has been derived, not too far from what may be naively expected from the antideuteron binding energy, i.e., $\sqrt{m_{p} B} \sim 46 \mathrm{MeV}$.

The differential probability with which an antiproton is produced during a proton-proton collision is related to the corresponding Lorentz invariant cross section through

$$
\sigma_{p-p}^{\mathrm{tot}} E_{\bar{p}} \mathcal{F}_{\bar{p}}\left(\sqrt{s, \overrightarrow{k_{\bar{p}}}}\right)=\left.E_{\bar{p}} \frac{d^{3} \sigma}{d^{3} \overrightarrow{k_{\bar{p}}}}\right|_{\mathrm{LI}} .
$$

The latter is experimentally well known. It is fairly well fitted by Tan and $\mathrm{Ng}$ 's parametrization [7] which has been used here. Assuming that the invariance of isospin holds, the antineutron production cross section is equal to its antiproton counterpart. The Lorentz invariant cross section for the production of antideuterons resulting from the impact of a highenergy cosmic-ray proton on a proton at rest has been derived by Chardonnet et al. [3] who showed that

$$
\begin{aligned}
& E_{\bar{D}} \frac{d^{3} \sigma_{\bar{D}}^{-}}{d^{3} \vec{k}_{\bar{D}}}=\left(\frac{m_{\bar{D}}^{-}}{m_{\bar{p}} m_{\bar{n}}}\right)\left(\frac{4}{3} \pi P_{\text {coal }}^{3}\right) \times \frac{1}{2 \sigma_{p-p}^{\text {tot }}} \\
& \times\left\{E_{\bar{p}} \frac{d^{3} \sigma_{\bar{p}}}{d^{3} \overrightarrow{k_{\bar{p}}}}\left(\sqrt{s, k_{\bar{p}}}\right) E_{\bar{n}} \frac{d^{3} \sigma_{\bar{n}}}{d^{3} \vec{k}_{\bar{n}}}\left(\sqrt{s}-2 E_{\bar{p}}, \overrightarrow{k_{\bar{n}}}\right)\right. \\
&\left.+\left(\overrightarrow{k_{\bar{p}} \leftrightarrow \vec{k}_{\bar{n}}}\right)\right\}
\end{aligned}
$$

The corresponding differential cross section obtains from the summation, in the galactic frame, of the Lorentz invariant production cross section (10)

$$
\frac{d \sigma_{p H \rightarrow \bar{D}}}{d E_{\bar{D}}^{-}}\left\{E_{p} \rightarrow E_{\bar{D}}\right\}=\left.2 \pi k_{\bar{D}} \int_{0}^{\theta_{\max }} E_{\bar{D}} \frac{d^{3} \sigma}{d^{3} \overrightarrow{k_{\bar{D}}}}\right|_{\mathrm{LI}} d(-\cos \theta) .
$$

In that frame, $\theta$ denotes the angle between the momenta of the incident proton and of the produced antideuteron. It is integrated up to a maximal value $\theta_{\max }$ set by the requirement that, in the center of mass frame of the reaction, the antideuteron energy $E_{\bar{D}}^{*}$ cannot exceed the bound

$$
E_{\bar{D}_{\max }^{*}}^{*}=\frac{s-16 m_{p}^{2}+m_{\bar{D}}^{2}}{2 \sqrt{s}} .
$$

The integral (11) is performed at fixed antideuteron energy $E_{\bar{D}}^{2}=m_{\bar{D}}^{2}+k_{\bar{D}}^{2}$.
Theoretical values for our coalescence momentum $P_{\text {coal }}$ range from $\sqrt{m_{p} B} \sim 46 \mathrm{MeV}$, naively derived from the antideuteron binding energy, up to $180 \mathrm{MeV}$, as would follow from a Hulthen parametrization of the deuterium wave function [8]. We therefore expect $P_{\text {coal }}$ to lie somewhere in the range between 50 and $200 \mathrm{MeV}$. Inside this range, since factorization might also involve an unknown coefficient that could be reabsorbed into $P_{\text {coal }}$, we have followed in Ref. [3] a fairly phenomenological approach. The coalescence momentum $P_{\text {coal }}$ has been determined directly by mere comparison between the accelerator data and our Lorentz invariant production cross section (10). Note that the antideuteron measurements have been performed at different center-ofmass energies and in various parts of phase space. The agreement with our naive scheme is surprisingly good given its crudeness. We therefore decided to subscribe to Occam's principle by keeping our antideuteron fusion model as simple as possible.

In the case of a neutralino annihilation, the differential multiplicity for antiproton production may be expressed as

$$
\frac{d N_{\bar{p}}}{d E_{\bar{p}}}=\sum_{F, h} B_{\chi h}^{(F)} \frac{d N_{\bar{p}}^{h}}{d E_{\bar{p}}} .
$$

The annihilation proceeds, through the various final states $F$, towards the quark or the gluon $h$ with the branching ratio $B_{\chi h}^{(F)}$. Quarks or gluons may be directly produced when a neutralino pair annihilates. They may alternatively result from the intermediate production of a Higgs or gauge boson as well as of a top quark. Each quark or gluon $h$ generates in turn a jet whose subsequent fragmentation and hadronization yields the antiproton energy spectrum $d N_{p}^{h} / d E_{p}^{-}$. Because neutralinos are at rest with respect to each other, the probability to form, say, an antiproton with momentum $\overrightarrow{k_{f}}$ is essentially isotropic

$$
\frac{d N_{\bar{p}}}{d E_{\bar{p}}}(\chi+\chi \rightarrow \bar{p}+\cdots)=4 \pi k_{\bar{p}} E_{\bar{p}} \mathcal{F}_{\bar{p}}\left(\sqrt{s}=2 m_{\chi}, E_{\bar{p}}\right) .
$$

Applying the factorization-coalescence scheme discussed above leads to the antideuteron differential multiplicity

$$
\frac{d N_{\bar{D}}^{-}}{d E_{\bar{D}}}=\left(\frac{4 P_{\mathrm{coal}}^{3}}{3 k_{\bar{D}}}\right)\left(\frac{m_{\bar{D}}^{-}}{m_{\bar{p}} m_{\bar{n}}}\right) \sum_{F, h} B_{\chi h}^{(F)}\left\{\frac{d N_{\bar{p}}^{h}}{d E_{\bar{p}}}\left(E_{\bar{p}}=E_{\bar{D}} / 2\right)\right\}^{2} .
$$

It may be expressed as a sum, extending over the various quarks and gluons $h$ as well as over the different annihilation channels $F$, of the square of the antiproton differential multiplicity. That sum is weighted by the relevant branching ratios. The antineutron and antiproton differential distributions have been assumed to be identical. The hypothesis that factorization holds is certainly conservative. We have naively assumed that both constituents of the antideuteron are independently isotropically distributed. That is certainly true for the first antinucleon and its associated jet. However, once the axis of the pair of jets is determined, the second anti- 
nucleon tends also to be aligned along that direction. Assuming that spherical symmetry holds in that case leads to underestimating the probability of fusion. If both antinucleons are back-to-back, they do not merge, as taken into account by our scheme. If they belong to the same jet, their angular correlation is stronger than what we have assumed, hence an enhanced probability of fusion. Our analysis is therefore meant to be conservative.

\section{THE DETECTION OF SPALLATION ANTIDEUTERONS}

As suggested by Parker, the propagation of cosmic rays inside the Galaxy is strongly affected by their scattering on the irregularities of magnetic fields. This results in a diffusive transport. In the following, we will assume an isotropic diffusion with an empirical value for the diffusion coefficient. Our Galaxy can be reasonably well modelled by a thin disk of atomic and molecular hydrogen, with radius $R$ $\sim 20 \mathrm{kpc}$ and thickness $\sim 200 \mathrm{pc}$. This gaseous ridge is sandwiched between two diffusion regions which act as confinement domains as a result of the presence of irregular magnetic fields. They extend vertically up to $\sim 3 \mathrm{kpc}$ apart from the central disk. That two-zone diffusion model is in good agreement with the observed primary and secondary nuclei abundances [9]. Notice that in the energetic range considered in our following analysis, the results provided by different propagation models are fairly close to each other. Choosing which of the models and its parameters should be favored in comparison with the others requires very detailed analysis and fits of the various light nuclei abundances. Such an effort is beyond the scope of the present paper.

Assuming a steady regime, the propagation of cosmic-ray antideuterons within the Milky Way is accounted for by the diffusion equation

$$
-K \Delta \psi_{\bar{D}}+\Gamma_{\bar{D}} \psi_{\bar{D}}+\frac{\partial}{\partial E}\left\{b(E) \psi_{\bar{D}}^{-}\right\}=q_{\bar{D}}^{\mathrm{sec}},
$$

where $\psi_{\bar{D}}$ is the density of antideuterons per unit of volume and per unit of energy.

In the left-hand side of relation (16), the first term describes the diffusion of the particles throughout the galactic magnetic fields. The coefficient $K$ is derived from measurements of the light element abundances in cosmic rays. It is constant at low energies, but beyond a critical value of $\mathcal{R}_{0}$ $=1 \mathrm{GV}$, it raises with rigidity $\mathcal{R}$ like

$$
K(\mathcal{R})=K_{0}\left(1+\frac{\mathcal{R}}{\mathcal{R}_{0}}\right)^{0.6}
$$

where $K_{0}=6 \times 10^{27} \mathrm{~cm}^{2} \mathrm{~s}^{-1}$. It is assumed to be essentially independent of the nature of the species that propagate throughout the Galaxy.

The second term accounts for the destruction of antideuterons through their interactions, mostly annihilations, with the interstellar medium. Antideuterons may also undergo fragmentation if they survive annihilation. In that case, they are broken apart as most of the cosmic-ray nuclei. The total collision rate is given by

$$
\Gamma_{\bar{D}}=\sigma_{\bar{D}_{H}} v_{\bar{D}} n_{\mathrm{H}},
$$

where $\sigma_{\bar{D}_{\mathrm{H}}}$ is the total antideuteron interaction cross section with protons, $v_{D}^{-}$denotes the velocity, and $n_{\mathrm{H}}=1 \mathrm{~cm}^{-3}$ is the average hydrogen density in the thin matter disk. That cross section has not been measured but the charged conjugate reaction, i.e., the collision of high-energy antiprotons on deuterium, has been observed and the relevant cross section may be found, for instance, in Ref. [10].

The last term in the left-hand side of relation (16) stands for the energy losses undergone by antideuterons as they diffuse in the galactic ridge. The rate $b\left(E_{\bar{D}}\right)=d E_{\bar{D}} / d t$ at which the antideuteron energy varies is essentially set by the ionization losses which the particle undergoes as it travels through interstellar gas. This mechanism yields the following contribution to the energy loss rate:

$b_{\text {ion }}\left(E_{D}^{-}\right)=-4 \pi r_{e}^{2} m_{e} c^{2} n_{\mathrm{H}} \frac{c}{\beta}\left\{\ln \left(\frac{2 m_{e} c^{2}}{E_{0}}\right)+\ln \left(\beta^{2} \gamma^{2}\right)-\beta^{2}\right\}$.

In molecular hydrogen, the ionization energy $E_{0}$ has been set equal to $19.2 \mathrm{eV}$; here $\gamma=E_{\bar{D}} / m_{\bar{D}}$. The classical radius of the electron is denoted by $r_{e}$ and the electron mass is $m_{e}$. In the case of antiprotons, it was realized $[7,4,5]$ that the dominant energy loss mechanism is actually their inelastic, but nonannihilating, interactions with interstellar protons. The latter are excited towards resonant states and hence absorb part of the antiproton energy. In the $\bar{p}$ frame, an incident proton kicks off the antiproton at rest, transferring some of its kinetic energy. In the case of antideuterons, however, such a process is no longer possible. In the antideuteron frame, any interaction which leads to an energy transfer $q^{0}$ larger than the binding energy $B$ would result in the destruction of the antinucleus. Actually, in the nonrelativistic regime at stake here, the energy transfer $q^{0}$ and momentum transfer $\vec{q}$ are related by

$$
q^{0} \simeq \frac{q^{2}}{2 m_{D}^{-}}
$$

The region which the interaction probes extends to a distance $\lambda \sim \hbar / q$. The proton will be sensitive to the antideuteron as a bulk nucleus only if $\lambda$ exceeds its size, i.e., $\sim 2 \mathrm{fm}$. This translates into $q \leqslant 100 \mathrm{MeV}$ and ultimately into $q^{0}$ $\leqslant 2.7 \mathrm{MeV}$. We can safely conclude that if the energy transfer $q^{0}$-as seen by the antideuteron in its rest frameexceeds a few $\mathrm{MeV}$, destruction occurs. The corresponding energy loss in the galactic frame where the proton, this time, is at rest is given by

$$
\delta T=\frac{m_{\bar{D}}}{m_{p}} q^{0}
$$


It cannot exceed $\sim 6 \mathrm{MeV}$. A larger value would be associated to a destructive energy transfer. We have also checked that the antideuteron does not survive an inelastic interaction during which the proton would be excited to a resonant state. Energy losses through elastic scatterings contribute a term

$$
b_{\text {scat }}\left(E_{\bar{D}}\right)=-\delta T\left\{\sigma_{\bar{D} \mathrm{H}}^{\mathrm{el}}\left(E_{\bar{D}}^{-}\right) n_{\mathrm{H}} v_{\bar{D}}^{-}\right\},
$$

where the elastic cross section $\sigma_{\bar{D} \mathrm{H}}^{\mathrm{el}}$ has been set equal to 10 $\mathrm{mb}$. Assuming a conservative value of $\delta T=10 \mathrm{MeV}$ leads to an increase of the antideuteron interstellar (IS) flux no larger than $6 \%$. The antideuteron, with its low binding energy of $B \sim 2.2 \mathrm{MeV}$, is actually an extremely fragile element. Interactions with the interstellar gas do not replenish the lowenergy tail of its spectrum but lead to its spallation. That is also why fragmentation dominates the interactions of cosmic-ray nuclei whose destructions occur at fixed energy per nucleon.

In the right-hand side of the diffusion Eq. (16), the production rate $q_{\bar{D}}^{\mathrm{sec}}$ of the spallation antideuterons involves a convolution over the incident cosmic-ray proton energy spectrum $\psi_{p}$ of the differential production cross sections (11)

$$
\begin{aligned}
q_{\bar{D}}^{\text {disk }}\left(E_{\bar{D}}\right)= & \int_{E_{\mathrm{th}}}^{+\infty} d E_{p} \psi_{p}\left(E_{p}\right) v_{p}\left\{n_{\mathrm{H}} \frac{d \sigma_{p \mathrm{H} \rightarrow \bar{D}}}{d E_{\bar{D}}}\right. \\
& \left.+n_{\mathrm{He}} \frac{d \sigma_{p \mathrm{He} \rightarrow \bar{D}}}{d E_{\bar{D}}}\right\}\left(E_{p} \rightarrow E_{\bar{D}}\right) .
\end{aligned}
$$

Interactions on hydrogen as well as on helium have been taken into account. The helium density $n_{\mathrm{He}}$ obtains from the numerical value of $n_{\mathrm{H}}=1 \mathrm{~cm}^{-3}$ and from the requirement that the helium mass fraction is $Y_{\mathrm{He}}=26 \%$. Whenever the proton sees the helium nucleus as a whole, particles tend to be produced in the rest frame of the target. That subthreshold effect is actually the dominant source of secondary antiprotons below $500 \mathrm{MeV}$. In order to take into account the production of antideuterons on interstellar helium, we have used the same recipe as in Ref. [5]. The interaction between an impinging proton with energy $E_{p}$ and a helium nucleus has the same effects as if the proton collided with a single nucleon at rest with the shifted energy

$$
E_{p}^{\mathrm{eff}}=E_{p}+0.6\left(E_{\mathrm{th}}-E_{p}\right)+1.1 \mathrm{GeV}
$$

for $E_{p}<E_{\text {th }}$ and with $E_{p}^{\mathrm{eff}}=E_{p}+1.1 \mathrm{GeV}$ otherwise. Production of an antinucleon below the threshold of $E_{\mathrm{th}}=7 m_{p}$ is therefore possible in the case of a helium target. For antideuterons, that subthreshold effect is no longer important. We find that the helium contribution amounts only to $\sim 10 \%$ of the entire low-energy $\bar{D}$ production. Because the formation of an antideuteron requires the manufacture of two antinucleon pairs, the energy of the impinging proton must exceed $17 m_{p}$, a region where the subthreshold effects mentioned above become negligible.

Relation (16) does not take into account diffusive reacceleration, a process that has been proposed in order to fit in a more natural way the cosmic-ray observations. If turbulent, the inhomogeneities of the magnetic field may behave as diffusion centers on which the high-energy particles bounce. This second order Fermi mechanism leads to the increase in time of the average energy of the cosmic rays. It also implies diffusion in energy space so that a monochromatic population spreads into a finite width distribution after some propagation. This effect may potentially flatten out the low-energy spectrum of secondary antiprotons and antideuterons. From a theoretical point of view, the variation of the diffusion coefficient $K$ with rigidity as $K \propto \mathcal{R}^{1 / 3}$ [11] comes from the hypothesis that the magnetic field fluctuations are turbulent and follow furthermore a Kolmogorov spectrum. This is strictly true if the interstellar gas is incompressible and homogeneously spread. Note also that the magnetic fluctuations are turbulent only in the absence of a mean field. Those three conditions are hardly met. The assumption that $K$ behaves as $\mathcal{R}^{1 / 3}$ is well motivated by aesthetics but is somewhat questionable. The real conditions of the interstellar medium do not particularly point toward that law [12]. Also from the experimental point of view, there is no clear indication that reacceleration takes place or is a necessary important ingredient of a propagation model. According to Ptuskin [13], cosmic ray measurements performed in the energy range between $100 \mathrm{MeV}$ and $100 \mathrm{GeV}$ do not imply the existence of diffusive reacceleration. On the other hand, the abundance of ${ }^{49} \mathrm{~V}$ and ${ }^{51} \mathrm{Cr}$, two secondary isotopes that are unstable through electron capture, indicate the possibility of a weak reacceleration with a modest energy increase not in excess of 100-200 MeV [14]. Finally, Heinbach and Simon have estimated the energy spectrum of light element cosmic rays [15] and of secondary antiprotons [16] under diffusive reacceleration. As shown in their Fig. 4, the flattening of the lowenergy antiproton spectrum is not as dramatic as may have been guessed initially once solar modulation is taken into account. Reacceleration leads to a factor of $\sim 4$ increase even at energies as low as $100 \mathrm{MeV}$. An effect of this size, which in principle could have some impact for antiprotons, would practically not change our results on antideuterons, because of the largely different behavior in the low-energy tail of primary versus secondary antideuterons. In conclusion, because the existence of a diffusive reacceleration in the manner of Kolmogorov is not supported by the observations and is not even well motivated from a theoretical point of viewsee above - we have decided to disregard this mechanism in our calculations. We feel that it should not be a strong source of flattening for the antideuteron low-energy spectrum. We nevertheless agree that in order to settle the question definitely, diffusive reacceleration should be taken into account in a complete numerical code which should also incorporate a large set of nuclear species, in particular the secondary nuclei that are unstable through electron capture. This is beyond the scope of the present analysis which was only meant to be exploratory.

The differential energy distribution $\psi_{\bar{D}}$ of secondary antideuterons is determined by solving Eq. (16). We have followed the standard approach which may be found in Ref. [17]. At the edge of the domain where the cosmic rays are confined, the particles escape freely, the diffusion becomes inefficient, and densities vanish. This provides the boundary 


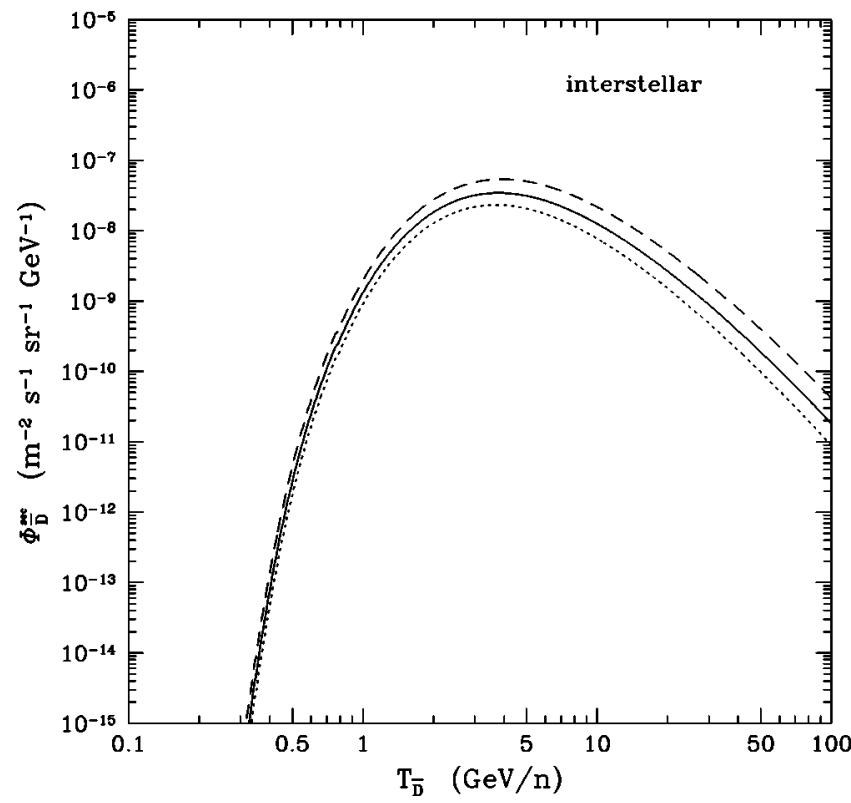

FIG. 1. The IS secondary flux of antideuterons, expressed in units of $\mathrm{m}^{-2} \mathrm{~s}^{-1} \mathrm{sr}^{-1} \mathrm{GeV}^{-1}$, is presented as a function of kinetic energy per nucleon. The solid curve corresponds to the median value of the cosmic-ray proton spectrum, as derived by Bottino et al. [4]. The dashed and dotted lines, respectively, stand for the maximal and minimal values of the primary proton flux from which the antideuterons originate.

conditions for solving Eq. (16). Then, because the problem is axisymmetric, the various cosmic-ray distributions may be expanded as a series of Bessel functions of zeroth order. Details may be found in Refs. $[4,18]$. The secondary antideuteron interstellar flux finally obtains from the differential energy spectrum

$$
\Phi_{\bar{D}}^{\mathrm{sec}}=\frac{1}{4 \pi} \psi_{\bar{D}}^{-} v_{\bar{D}}^{-}
$$

The IS flux of spallation antideuterons is presented in Fig. 1 as a function of the kinetic energy per nucleon. As explained in Bottino et al. [4], the IS proton flux is still uncertain around $\sim 20-100 \mathrm{GeV}$, an energy range that contributes most to the integral (23). We have borrowed the parametrization

$$
\Phi_{p}^{\mathrm{IS}}=A \beta\left(\frac{E_{p}}{1 \mathrm{GeV}}\right)^{-\alpha}
$$

The median IS proton flux corresponds to a normalization factor of $A=15950$ protons $\mathrm{m}^{-2} \mathrm{~s}^{-1} \mathrm{sr}^{-1} \mathrm{GeV}^{-1}$ with a spectral index of $\alpha=2.76$. The normalization factor $A$ has been varied from 12300 (minimal) up to 19600 protons $\mathrm{m}^{-2} \mathrm{~s}^{-1} \mathrm{sr}^{-1} \mathrm{GeV}^{-1}$ (maximal). Accordingly, the minimal and maximal IS proton fluxes, respectively, correspond to the spectral indices $\alpha=2.61$ and 2.89. In Fig. 1, the solid curve features the IS secondary antideuterons generated from the median proton spectrum. The maximal (dashed line) and minimal (dotted line) distributions delineate the band within which the spallation antideuteron signal lies. The flux

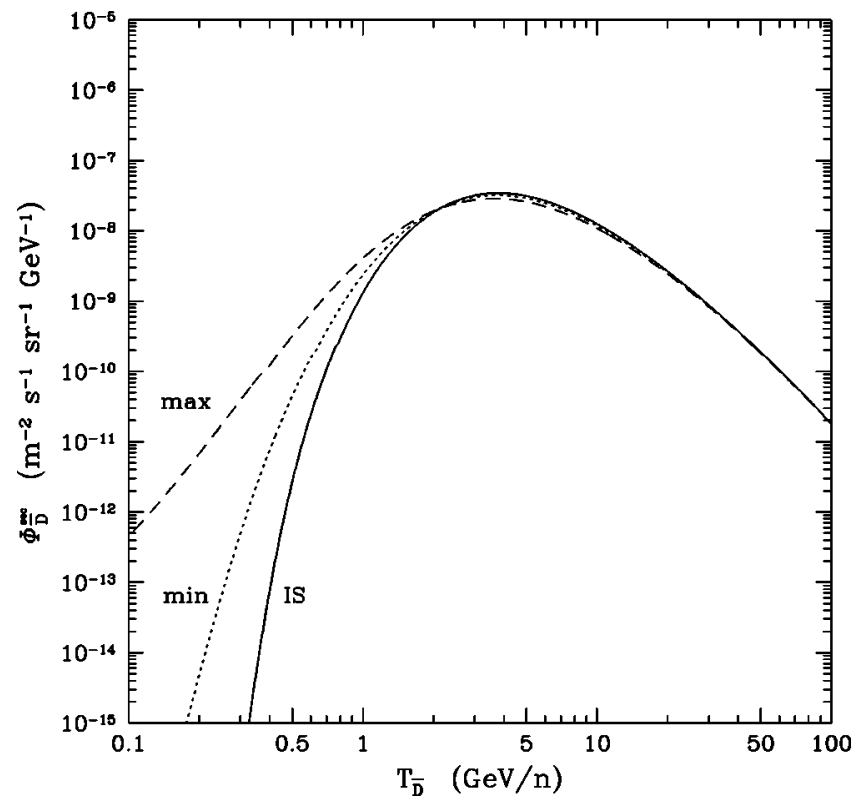

FIG. 2. The median IS spectrum of Fig. 1 (solid curve) has been modulated at solar maximum (dashed line) and minimum (dotted line).

reaches a maximum value comprised between 2.1 and $4.9 \times 10^{-8} \bar{D} \mathrm{~m}^{-2} \mathrm{~s}^{-1} \mathrm{sr}^{-1} \mathrm{GeV}^{-1}$ for a kinetic energy of $\sim 4 \mathrm{GeV} / n$. The antideuteron spectrum sharply drops below a few $\mathrm{GeV} / n$. Remember that in the galactic frame, the production threshold is $17 m_{p}$. When a high-energy cosmic-ray proton impinges on a hydrogen atom at rest, the bulk of the resulting antiprotons and antineutrons keep moving, with kinetic energies $\sim 10-20 \mathrm{GeV}$. For kinematical reasons, the production of antinucleons at rest with respect to the Galaxy is extremely unprobable. The manufacture of a low-energy antideuteron is even more unprobable. It actually requires the creation of both an antiproton and an antineutron at rest. The momenta need to be aligned in order for fusion to succesfully take place. Low-energy antideuterons produced as secondaries in the collisions of high-energy cosmic rays with the interstellar material are therefore extremely scarce, with a completely depleted energy spectrum below $\sim 1 \mathrm{GeV} / n$. Energy losses tend to shift the antideuteron spectrum towards lower energies with the effect of replenishing the low-energy tail with the more abundant species which, initially, had a higher energy. This process tends to slightly soften the strong decrease of the low-energy antideuteron spectrum. The effect is nevertheless mild. Remember that in the case of antiprotons, it is actually the inelastic but nonannihilating interactions which considerably flatten the $\bar{p}$ distribution. Ionization losses are not enough to significantly affect the energy spectrum. The IS secondary antideuterons are therefore extremely depleted below $\sim 1 \mathrm{GeV} / n$. The spallation background is negligible in the region where supersymmetric $\bar{D}$ 's are expected to be most abundant. This feature makes the detection of low-energy antideuterons an interesting signature of the presence of supersymmetric relics in the Galaxy.

In Fig. 2, the median IS $\bar{D}$ spectrum (solid curve) has 
been modulated at solar maximum (dashed line) and solar minimum (dotted line). We have applied the forced field approximation [19] to estimate the effect of the solar wind on the cosmic-ray energies and fluxes. For the energies at stake, this amounts to simply shifting the IS energy of a nucleus $N$, with charge $Z$ and atomic number $A$, by a factor of $Z e \Phi$. The solar modulation parameter $\Phi$ has the same dimensions as a rigidity or an electric potential. The Earth $(\oplus)$ and IS energies, per nucleon, are therefore related by

$$
E_{N}^{\oplus} / A=E_{N}^{\mathrm{IS}} / A-|Z| e \Phi / A .
$$

In Perko's approximation, antinuclei are affected in just the same way as nuclei. Their energy decreases as they penetrate the heliomagnetic field. Once the momenta at the Earth $p_{N}^{\oplus}$ and at the boundaries of the heliosphere $p_{N}^{\text {IS }}$ are determined, the flux modulation ensues

$$
\frac{\Phi_{N}^{\oplus}\left(E_{N}^{\oplus}\right)}{\Phi_{N}^{\mathrm{IS}}\left(E_{N}^{\mathrm{IS}}\right)}=\left\{\frac{p_{N}^{\oplus}}{p_{N}^{\mathrm{IS}}}\right\}^{2} .
$$

Antideuterons undergo an energy loss, per nucleon, half that of protons and antiprotons. At solar minimum (maximum) the modulation parameter $\Phi$ has been set equal to $320 \mathrm{MV}$ (800 MV) [4]. The energy shift is larger at solar maximum than at solar minimum. Once modulated, the sharply decreasing IS antideuteron distribution tends to be flatter at solar maximum as is clear in Fig. 2. We estimate that a total of 12-13 secondary antideuterons may be collected by the AMS Collaboration during the space station stage, in the energy range extending up to $100 \mathrm{GeV} / n$. These antideuterons correspond to IS energies in excess of $\sim 3 \mathrm{GeV} / n$, a region free from the effects of solar modulation. This result takes into account the geomagnetic suppression as discussed in Sec. V.

As estimated in Ref. [4], the uncertainty of the modulation parameter $\Phi$ does not exceed $\sim 50 \mathrm{MeV}$. At low energies, this implies an error on the measured flux of order

$$
\frac{\delta \Phi_{N}^{\oplus}}{\Phi_{N}^{\oplus}} \simeq \frac{|Z| e \delta \Phi}{T_{N}^{\oplus}} .
$$

We infer an uncertainty of $\sim 25 \%$ on the antideuteron flux at Earth for a kinetic energy of $100 \mathrm{MeV} / n$, decreasing to $\sim 5 \%$ at $500 \mathrm{MeV} / n$. Because the geomagnetic cutoff prevents low-energy particles from reaching a satellite-borne detector, uncertainties in the solar modulation will only mildly affect our estimates of the number of primary supersymmetric antideuterons which AMS on board ISS may potentially collect.

\section{THE SUPERSYMMETRIC ANTIDEUTERON SIGNAL}

As a theoretical framework, we use the minimal supersymmetric extension of the standard model (MSSM) [20], which conveniently describes the supersymmetric phenomenology at the electroweak scale, without too strong theoretical assumptions. This model has been largely adopted by many authors for evaluations of the neutralino relic abun- dance and detection rates (for reviews, see Refs. [21],[22]).

The MSSM is defined at the electroweak scale as a straightforward supersymmetric extension of the standard model. The Higgs sector consists of two Higgs doublets $H_{1}$ and $\mathrm{H}_{2}$ and, at the tree level, is fully described by two free parameters, namely: the ratio of the two vacuum expectation values $\tan \beta \equiv\left\langle H_{2}\right\rangle /\left\langle H_{1}\right\rangle$ and the mass of one of the three neutral physical Higgs fields, which we choose to be the mass $m_{A}$ of the neutral pseudoscalar one. Once radiative corrections are introduced, the Higgs sector depends also on the squark masses through loop diagrams. The radiative corrections to the neutral and charged Higgs bosons, adopted in the present paper, are taken from Refs. [23],[24]. The other parameters of the model are defined in the superpotential, which contains all the Yukawa interactions and the Higgsmixing term $\mu H_{1} H_{2}$, and in the soft-breaking Lagrangian, which contains the trilinear and bilinear breaking parameters and the soft gaugino and scalar mass terms. In this model, the neutralino is defined as the lowest-mass linear superposition of photino $(\widetilde{\gamma}), Z$-ino $(\widetilde{Z})$, and the two Higgsino states $\left(\widetilde{H}_{1}^{0}, \widetilde{H}_{2}^{0}\right)$,

$$
\chi \equiv \alpha_{1} \widetilde{\gamma}+a_{2} \widetilde{Z}+a_{3} \widetilde{H}_{1}^{0}+a_{4} \widetilde{H}_{2}^{0} .
$$

In order to deal with manageable models, it is necessary to introduce some assumptions which establish relations among the too many free parameters at the electroweak scale. We adopt the following usual conditions. All trilinear parameters are set to zero except those of the third family, which are unified to a common value $A$. All squark and slepton softmass parameters are taken as degenerate: $m_{\tilde{l}_{i}}=m_{\tilde{q}_{i}} \equiv m_{0}$. The gaugino masses are assumed to unify at $M_{\mathrm{GUT}}$, and this implies that the $U(1)$ and $S U(2)$ gaugino masses are related at the electroweak scale by $M_{1}=(5 / 3) \tan ^{2} \theta_{W} M_{2}$. When all these conditions are imposed, the supersymmetric parameter space is completely described by six independent parameters, which we choose to be $M_{2}, \mu, \tan \beta, m_{A}, m_{0}, A$. In our analyses, we vary them in the following ranges: $20 \mathrm{GeV}$ $\leqslant M_{2} \leqslant 500 \mathrm{GeV} ; 20 \mathrm{GeV} \leqslant|\mu| \leqslant 500 \mathrm{GeV} ; \quad 80 \mathrm{GeV} \leqslant m_{A}$ $\leqslant 1000 \mathrm{GeV} ; 100 \mathrm{GeV} \leqslant m_{0} \leqslant 1000 \mathrm{GeV} ; \quad-3 \leqslant A \leqslant+3 ; 1$ $\leqslant \tan \beta \leqslant 50$.

The supersymmetric parameter space is constrained by all the experimental limits achieved at accelerators on supersymmetric and Higgs searches [25]. Also the constraints due to the $b \rightarrow s+\gamma$ process $[26,27]$ have been taken into account (see Ref. [28] for a discussion of our implementation of the $b \rightarrow s+\gamma$ constraint and for the relevant references). We further require the neutralino to be the lightest supersymmetric particle (LSP) and the supersymmetric configurations to provide a neutralino relic abundance in accordance with the cosmological bound $\Omega_{\chi} h^{2} \leqslant 0.7$ [21].

For the evaluation of the averaged annihilation cross section $\left\langle\sigma_{\mathrm{ann}} v\right\rangle$, we have followed the procedure outlined in Ref. [29]. We have considered all the tree-level diagrams which are responsible for neutralino annihilation and which are relevant to $\bar{p}$ production, namely: annihilation into quarkantiquark pairs, into gauge bosons, into a Higgs boson pair, and into a Higgs and a gauge boson. For each final state we 
have considered all the relevant Feynman diagrams, which involve the exchange of Higgs and $Z$ bosons in the $s$-channel and the exchange of squarks, neutralinos, and charginos in the $t$ and $u$ channels. Finally, we have included the one-loop diagrams which produce a two-gluon final state [30]. The $\bar{p}$ differential distribution $d N_{\bar{p}} / d E_{\bar{p}}$ has been evaluated as discussed in Ref. [29]. Here we only recall that we have calculated the branching ratios $B_{\chi h}^{(F)}$ for all annihilation final states $F$ which may produce $\bar{p}$ 's. These final states fall into two categories: (i) direct production of quarks and gluons and (ii) generation of quarks through the intermediate production of Higgs bosons, gauge bosons, and $t$ quark. In order to obtain the distributions $d N_{\bar{p}}^{h} / d E_{\bar{p}}$, the hadronization of quarks and gluons has been computed by using the Monte Carlo code JETSET 7.2 [31], introducing negligible uncertainties-for a more detailed analysis, see Ref. [5]. For the top quark, we have considered it to decay before hadronization. The source term for supersymmetric antideuterons

$$
q_{\bar{D}}^{\text {susy }}(\chi+\chi \rightarrow \bar{D}+\cdots)=\left\langle\sigma_{\mathrm{ann}} v\right\rangle \frac{d N_{\bar{D}}^{-}}{d E_{\bar{D}}^{-}}\left\{\frac{\rho_{\chi}}{m_{\chi}}\right\}^{2}
$$

supplements the spallation contribution $q_{\bar{D}}^{\text {sec }}$ in the diffusion Eq. (16). The propagation of primary antideuterons from the remote regions of the galactic halo to the Earth has been treated as explained in Ref. [4]. The neutralino distribution has been assumed to be spherical, with radial dependence

$$
\rho_{\chi}=\rho_{\chi}^{\odot}\left\{\frac{a^{2}+r_{\odot}^{2}}{a^{2}+m^{2}}\right\},
$$

where $m^{2}=r^{2}+z^{2}$. The solar system is at a distance $r_{\odot}$ of 8 $\mathrm{kpc}$ from the galactic center. The dark matter halo has a core radius $a=3.5 \mathrm{kpc}$ and its density in the solar neighborhood is $\rho_{\chi}^{\odot}=0.4 \mathrm{GeV} \mathrm{cm}^{-3}$ [21]. These parameters are known with some uncertainties. In particular, the local density may lay in the range $0.1<\rho_{\chi}^{\odot}<0.7 \mathrm{GeV} \mathrm{cm}^{-3}$, implying strong variations in the antideuteron flux. The latter depends on the square of that density. Clumpiness may also significantly enhance the signal.

In Fig. 3, both primary (supersymmetric) and secondary (spallation) interstellar antideuteron energy spectra are presented. The secondary flux (heavier solid line) drops sharply at low energies as discussed above. The four supersymmetric examples of Table I are respectively featured by the solid (a), dotted (b), dashed (c), and dot-dashed (d) curves. The corresponding primary fluxes flatten at low energy where they reach a maximum. As the secondary $\bar{D}$ background vanishes, the supersymmetric signal is the largest. Neutralino annihilations actually take place at rest in the galactic frame. The fragmentation and subsequent hadronization of the jets at stake tend to favor the production of low-energy species. Therefore, the spectrum of supersymmetric antiprotons-and antineutrons-is fairly flat below $\sim 1 \mathrm{GeV}$. For the same reasons, the coalescence of the primary antideuterons produced in neutralino annihilations predominantly takes place with the two antinucleons at rest, hence a flat spectrum at low energy, as is clear in Fig. 3. The fusion of an antideu-

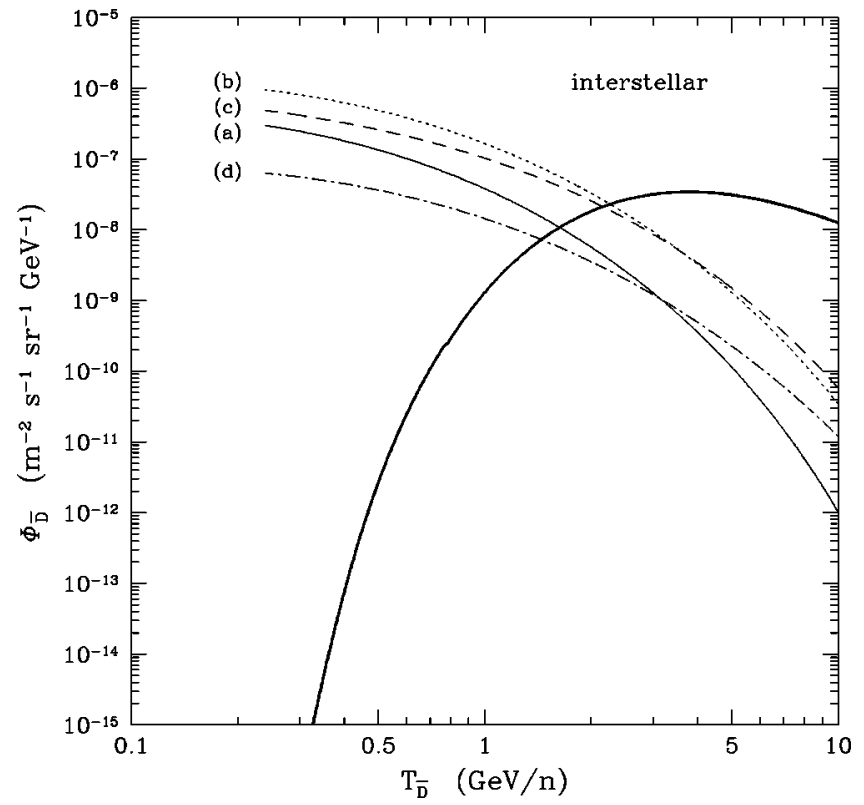

FIG. 3. The IS flux of secondary antideuterons (heavier solid curve) decreases at low energy whereas the energy spectrum of the antideuterons from supersymmetric origin tends to flatten. The four cases of Table I are respectively featured by the solid (a), dotted (b), dashed (c), and dot-dashed (d) curves.

teron requires actually that its antinucleon constituents should be aligned in momentum space. Consequently, secondary antideuterons are completely depleted below $\sim 1$ $\mathrm{GeV}$ while the primary species are mostly produced in that low-energy regime. This trend still appears once the energies and fluxes are modulated. The (a) and (b) panels of Fig. 4, respectively, show the effects of solar modulation at maximum and minimum. The spallation background somewhat flattens. It is still orders of magnitude below the supersymmetric signal which clearly exhibits a plateau.

It is difficult to establish a correlation between the $\bar{D}$ flux and the neutralino mass. In case (c), for instance, $m_{\chi}$ is $\sim 3$ times larger than in case (a) and yet the corresponding antideuteron flux is larger. It is not obvious either that gauginolike mixtures lead to the largest $\bar{D}$ signals. Table I gives a flavor of the complexity and of the richness of the supersymmetric parameter space.

In Fig. 5, the supersymmetric-to-spallation IS flux ratios for antiprotons (lower curves) and antideuterons (upper curves) are presented as a function of the kinetic energy per nucleon. In the case of antiprotons, the primary-to-secondary ratio is much smaller than for antideuterons. For the configurations of Table I presented here, the $\bar{p}$ primary flux is at the same level as the spallation background. The supersymmetric antiproton signal is swamped in the flux of the secondaries. This is not the case for antideuterons. At low energies, their supersymmetric flux is several orders of magnitude above background. Antideuterons appear therefore as a much cleaner probe of the presence of supersymmetric relics in the galactic halo than antiprotons. The price to pay, however, is a much smaller flux. Typical $\bar{D}$ spectra may reach up to $10^{-6}-10^{-5} \mathrm{~m}^{-2} \mathrm{~s}^{-1} \mathrm{sr}^{-1} \mathrm{GeV}^{-1}$. This corresponds to an an- 
TABLE I. These four cases illustrate the richness of the supersymmetric parameter space. There is no obvious correlation between the antiproton and antideuteron Earth fluxes with the neutralino mass $m_{\chi}$. Case (c) is a gaugino-Higgsino mixture and still yields signals comparable to those of case (a), yet a pure gaugino. Antiduteron fluxes are estimated at both solar minimum and maximum, for a modulated energy of 0.24 $\mathrm{GeV} / n$. The last column features the corresponding number of $\bar{D}$ 's which AMS on board ISSA can collect below $3 \mathrm{GeV} / n$.

\begin{tabular}{cccccccc}
\hline \hline Case & $m_{\chi}$ & $P_{g}(\%)$ & $\Omega_{\chi} h^{2}$ & $\Phi_{\bar{p}}^{\min }(0.24 \mathrm{GeV})$ & $\Phi_{\bar{D}}^{\min }(0.24 \mathrm{GeV} / n)$ & $\Phi_{\bar{D}}^{\max }(0.24 \mathrm{GeV} / n)$ & $N_{\bar{D}}^{\max }$ \\
\hline $\mathrm{a}$ & 36.5 & 96.9 & 0.20 & $1.2 \times 10^{-3}$ & $1.0 \times 10^{-7}$ & $2.9 \times 10^{-8}$ & 0.6 \\
$\mathrm{~b}$ & 61.2 & 95.3 & 0.13 & $3.9 \times 10^{-3}$ & $3.5 \times 10^{-7}$ & $1.1 \times 10^{-7}$ & 2.9 \\
$\mathrm{c}$ & 90.4 & 53.7 & 0.03 & $1.1 \times 10^{-3}$ & $1.8 \times 10^{-7}$ & $6.1 \times 10^{-8}$ & 2.0 \\
$\mathrm{~d}$ & 120 & 98.9 & 0.53 & $2.9 \times 10^{-4}$ & $2.5 \times 10^{-8}$ & $8.6 \times 10^{-9}$ & 0.3 \\
\hline \hline
\end{tabular}
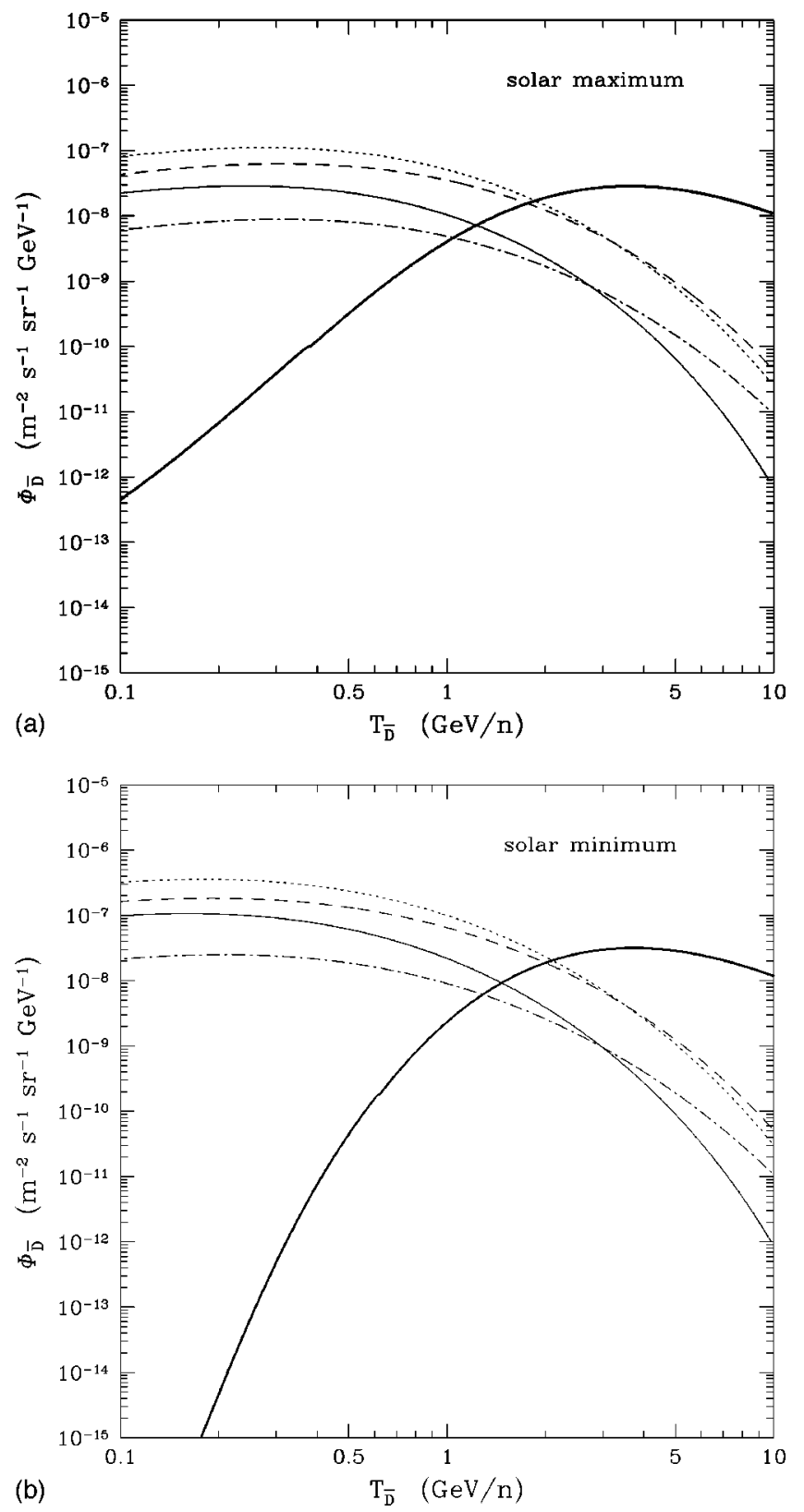

FIG. 4. Same as in Fig. 3 but modulated at solar maximum (a) and minimum (b). tiproton signal of $10^{-2}-10^{-1} \mathrm{~m}^{-2} \mathrm{~s}^{-1} \mathrm{sr}^{-1} \mathrm{GeV}^{-1}$, i.e., four orders of magnitude larger. It is therefore crucial to ascertain which portion of the supersymmetric configurations will be accessible to future experiments through the detection of low-energy cosmic-ray antideuterons.

\section{DISCUSSION AND CONCLUSIONS}

In order to be specific, we have estimated the amount of antideuterons which may be collected by the AMS experiment once it is on board ISSA. The future space station is scheduled to orbit at $400 \mathrm{~km}$ above sea level, with an inclination of $\alpha=52^{\circ}$ with respect to the Earth's equator. A revolution takes about $1.5 \mathrm{~h}$ so that ISSA should fly over the

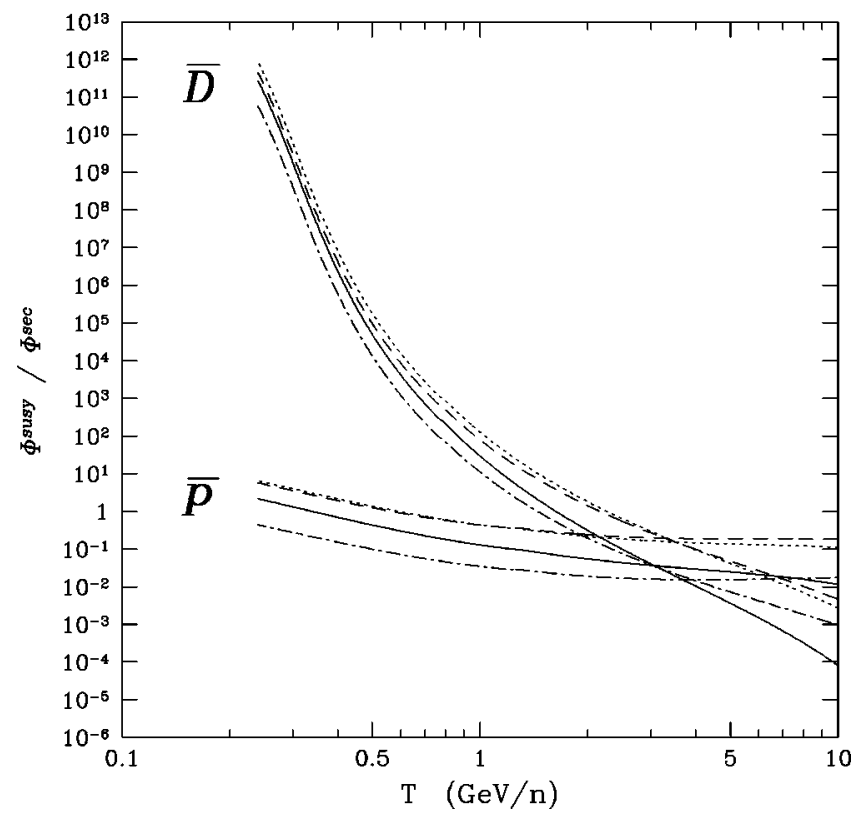

FIG. 5. The supersymmetric-to-secondary IS flux ratio for antiprotons (lower curves) and antideuterons (upper curves) is presented as a function of the kinetic energy per nucleon. The supersymmetric configurations are those reported in Table I and featured in Figs. 3 and 4. Below a few $\mathrm{GeV} / n$, the flux ratio is always larger for $\bar{D}$ 's than for $\bar{p}$ 's. For the supersymmetric configurations of Table I, the antiproton signal is swamped into its background. This is not the case for antideuterons. At low energy, the flux of primaries is several orders of magnitude above the $\bar{D}$ background. 
same spot every day. The AMS detector may be pictured as a cylindrical magnetic field with diameter $D=110 \mathrm{~cm}$. At any time, its axis points towards the local vertical direction. The colatitude of the north magnetic pole has been set equal to $Y=11^{\circ}$. At any given time $t$ along the orbit, the geomagnetic latitude $\varrho$ of ISSA may be inferred from

$$
\begin{aligned}
\sin \varrho= & \sin \Upsilon \cos \left(\Omega_{\mathrm{sid}} t\right) \cos \left(\Omega_{\mathrm{orb}} t+\varphi\right) \\
& +\cos \alpha \sin \Upsilon \sin \left(\Omega_{\mathrm{sid}} t\right) \sin \left(\Omega_{\mathrm{orb}} t+\varphi\right) \\
& +\sin \alpha \cos \Upsilon \sin \left(\Omega_{\mathrm{orb}} t+\varphi\right),
\end{aligned}
$$

where $\Omega_{\text {sid }}$ and $\Omega_{\text {orb }}$, respectively, denote the angular velocities associated to the sidereal rotation of the Earth and to the orbital motion of the space station. The phase $\varphi$ depends on the orbital initial conditions and does not affect the result if a large number of revolutions - typically 100-is considered. The Earth is shielded from cosmic rays because its magnetic field prevents particles from penetrating downwards. At any given geomagnetic latitude $\varrho$, there exists a rigidity cutoff $\mathcal{R}_{\text {min }}$ below which the cosmic-ray flux is suppressed. This lower bound depends on the radius $R$ of the orbit through

$$
\mathcal{R}_{\min }=\frac{\mu_{\oplus}}{R^{2}} \frac{\cos ^{4} \varrho}{\varpi^{2}},
$$

where $\mu_{\oplus}$ denotes the Earth's magnetic dipole moment and $\mu_{\oplus} / R_{\oplus}^{2} \simeq 60 \mathrm{GV}$. The term wstands for

$$
\varpi=1+\sqrt{1+\cos \theta \cos ^{3} \varrho} .
$$

It depends on the angle $\theta$ between the cosmic-ray momentum at the detector and the local east-west line that points in the orthoradial direction of an axisymmetric coordinate system. Notice that because we are interested here in singly charged species, the rigidity amounts to the momentum $p$. Once the cosmic-ray energy as well as the geomagnetic latitude are specified, the solid angle $\Omega_{\text {cut }}$ inside which the direction of the incoming particle lies may be derived from relations (34) and (35). The AMS detector looks upwards within $\sim 27^{\circ}$ around the vertical. This corresponds to a solid angle of $\Omega_{\text {det }}=0.68$ sr. Because the apparatus does not point towards the local east or west, impinging particles may not be seen by the instrument. The effective solid angle $\Omega_{\text {eff }}$ through which they are potentially detectable corresponds to the overlap, if any, between $\Omega_{\text {cut }}$ and $\Omega_{\text {det }}$. The value of $\Omega_{\text {eff }}$ depends on the cosmic-ray rigidity $p$ as well as on the precise location of the detector along the orbit. The detector acceptance may therefore be defined as

$$
\aleph(p)=\frac{\pi}{4} D^{2} \int \Omega_{\mathrm{eff}}(p, t) d t,
$$

where the time integral runs over the duration $\tau$ of the space mission. In the case of AMS on board ISSA, $\tau$ is estimated to be $10^{8} \mathrm{~s}$ ( $3 \mathrm{yrs}$ ). Between $100 \mathrm{MeV} / n$ and $100 \mathrm{GeV} / n$, we infer a total acceptance of $5.8 \times 10^{9} \mathrm{~m}^{2} \mathrm{~s} \mathrm{sr} \mathrm{GeV}$ for antiprotons and of $6 \times 10^{9} \mathrm{~m}^{2} \mathrm{~s} \mathrm{srGeV}$ for antideuterons. The net number of cosmic-ray species which AMS may collect on

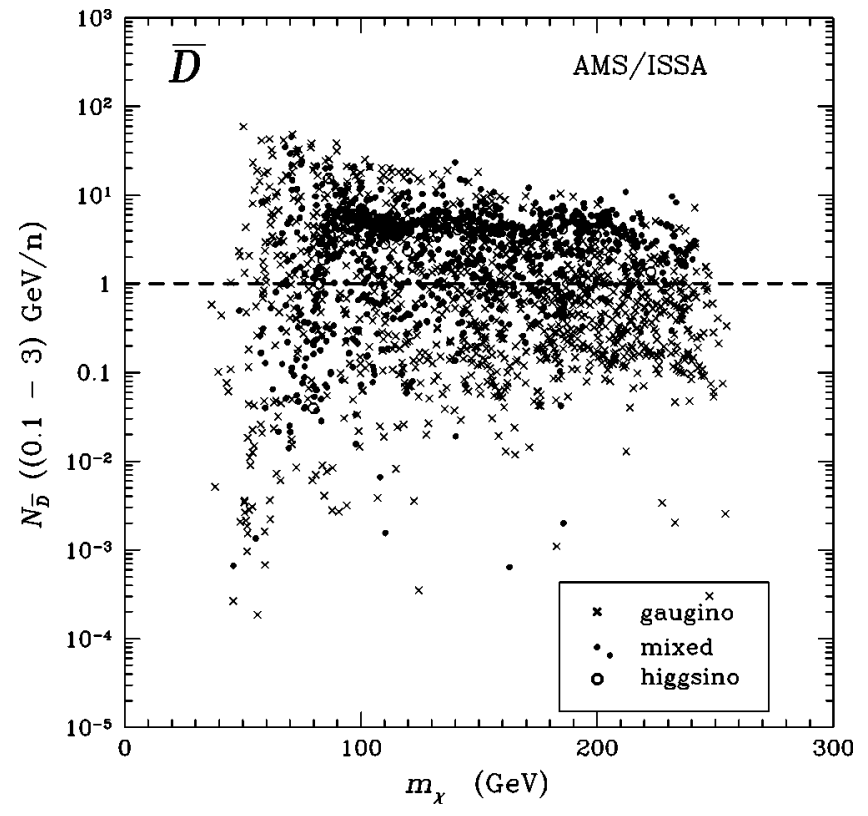

FIG. 6. The supersymmetric $\bar{D}$ flux has been integrated over the range of IS energies extending from 0.1 up to $3 \mathrm{GeV} / n$. The resulting yield $N_{\bar{D}}$ of antideuterons which AMS on board ISSA can collect is plotted as a function of the neutralino mass $m_{\chi}$. Modulation has been considered at solar maximum.

board ISSA is actually a convolution of the detector acceptance with the relevant differential flux at Earth. For antideuterons, this leads to

$$
N_{\bar{D}}^{-}=\int \aleph\left(p_{\bar{D}}^{\oplus}\right) \Phi_{\bar{D}}^{\oplus} d T_{\bar{D}}^{\oplus},
$$

where the integral runs on the $\bar{D}$ modulated energy $T_{\bar{D}}^{\oplus}$.

Integrating the secondary flux discussed in Sec. III leads respectively to a total of 12.3 and 13.4 antideuterons, depending on whether the solar cycle is at maximum or minimum. These spallation $\bar{D}$ 's are mostly expected at high energies. As is clear from Figs. 3 and 4, the secondary flux drops below the supersymmetric signal below a few $\mathrm{GeV} / n$. The transition typically takes place for an interstellar energy of $3 \mathrm{GeV} / n$. Below that value, the secondary antideuteron signal amounts to a total of only 0.6 (solar maximum) and 0.8 (solar minimum) nuclei. Most of the supersymmetric signal is therefore concentrated in a low-energy band extending from the AMS threshold of $100 \mathrm{MeV} / n$ up to a modulated energy of $2.6 \mathrm{GeV} / n$ (maximum) or $2.84 \mathrm{GeV} / n$ (minimum) which corresponds to an upper bound of $3 \mathrm{GeV} / n$ in interstellar space. In this low-energy region where spallation antideuterons yield a negligible background, the AMS acceptance is $2.2 \times 10^{7} \mathrm{~m}^{2} \mathrm{~s} \mathrm{sr} \mathrm{GeV}$ for antiprotons and $5.5 \times 10^{7} \mathrm{~m}^{2} \mathrm{~s} \mathrm{sr} \mathrm{GeV}$ for antideuterons.

For each supersymmetric configuration, the $\bar{D}$ flux has been integrated over that low-energy range. The resulting yield $N_{\bar{D}}$ which AMS may collect on board ISSA is presented as a function of the neutralino mass $m_{\chi}$ in the scatter plot of Fig. 6. During the AMS mission, the solar cycle will be at maximum. Most of the configurations are gaugino like 
(crosses) or mixed combinations of gaugino and Higgsino states (dots). A significant portion of the parameter space is associated to a signal exceeding one antideuteronhorizontal dashed line. In a few cases, AMS may even collect more than a dozen low-energy $\bar{D}$ nuclei. However, when the antideuteron signal exceeds $\sim 20$ particles, the associated antiproton flux is larger than what BESS95+97 [32] has measured.

The scatter plot of Fig. 6 may be translated into a limit on the antideuteron flux $\Phi_{\bar{D}}^{\oplus}$ at the Earth. Table I gives a flavor of the relation between that flux and the yield $N_{\bar{D}}$ of low-energy antideuterons. At solar maximum, a value of $N_{\bar{D}}^{-}=1$ translates, on average, into a flux of $\sim 3.2 \times 10^{-8} \bar{D} \mathrm{~m}^{-2} \mathrm{~s}^{-1} \mathrm{sr}^{-1} \mathrm{GeV}^{-1}$ for a modulated energy of $240 \mathrm{MeV} / n$. The energy spectrum matters of course. For the steep differential flux of case (a), a value of $4.8 \times 10^{-8} \bar{D} \mathrm{~m}^{-2} \mathrm{~s}^{-1} \mathrm{sr}^{-1} \mathrm{GeV}^{-1}$ is necessary in order to achieve a signal of at least one antideuteron. In case (d) where the spectrum is much flatter, the same $\bar{D}$ yield is reached for a flux of only $2.8 \times 10^{-8} \bar{D}$ $\mathrm{m}^{-2} \mathrm{~s}^{-1} \mathrm{sr}^{-1} \mathrm{GeV}^{-1}$. The horizontal dashed lines of Figs. 7 should therefore be understood as averaged limits. They are nevertheless indicative of the level of sensitivity which may be reached through the search for low-energy antideuterons. The (a) and (b) panels, respectively, correspond to a solar activity taken at maximum and minimum. In these scatter plots, the $\bar{D}$ modulated flux is featured as a function of the neutralino mass $m_{x}$. The antideuteron energy at the Earth has been set equal to $240 \mathrm{MeV} / n$. The flux $\Phi_{\bar{D}}^{\oplus}$ is larger at solar minimum - when modulation is weaker - than at maximum. The lower the cosmic-ray energy, the larger that effect. The plateaux of Figs. 4 illustrate the flatness of the supersymmetric $\bar{D}$ spectra at low energies. These plateaux actually exhibit a shift by a factor $\sim 3$ between the left and right panels. Accordingly, the constellation of supersymmetric configurations in Figs. 7 is shifted upwards, by the same amount, between solar maximum (left panel) and minimum (right panel). At larger energies, the variation of the flux at Earth during the solar cycle is milder. Above a few $\mathrm{GeV} / n$, solar modulation has no effect. The number of supersymmetric antideuterons collected at low energy obtains from the convolution of Eq. (37). It also varies during the solar cycle, in a somewhat lesser extent, however, than the abovementioned plateaux. Between maximum and minimum, the value of $N_{\bar{D}}$ only varies by a factor of $\sim 2$, to be compared to a flux increase of $\sim 3$. At solar maximum, when AMS-ISSA will be operating, a signal of one antideuteron translates into a flux sensitivity of $\sim 3.2 \times 10^{-8}$ antinuclei $\mathrm{m}^{-2} \mathrm{~s}^{-1} \mathrm{sr}^{-1} \mathrm{GeV}^{-1}$. At minimum, the same signal would translate into the weaker limit of $\sim 4.8 \times 10^{-8}$ antideuterons $\mathrm{m}^{-2} \mathrm{~s}^{-1} \mathrm{sr}^{-1} \mathrm{GeV}^{-1}$ and the horizontal dashed line is shifted upwards by $\sim 50 \%$. The supersymmetric configurations which an antideuteron search may unravel are nevertheless more numerous at solar minimum. Between the (a) and the (b) panels, the constellation of representative points is actually shifted upwards and, relative to the limit of sensitivity, the increase amounts to a factor $\sim 2$.
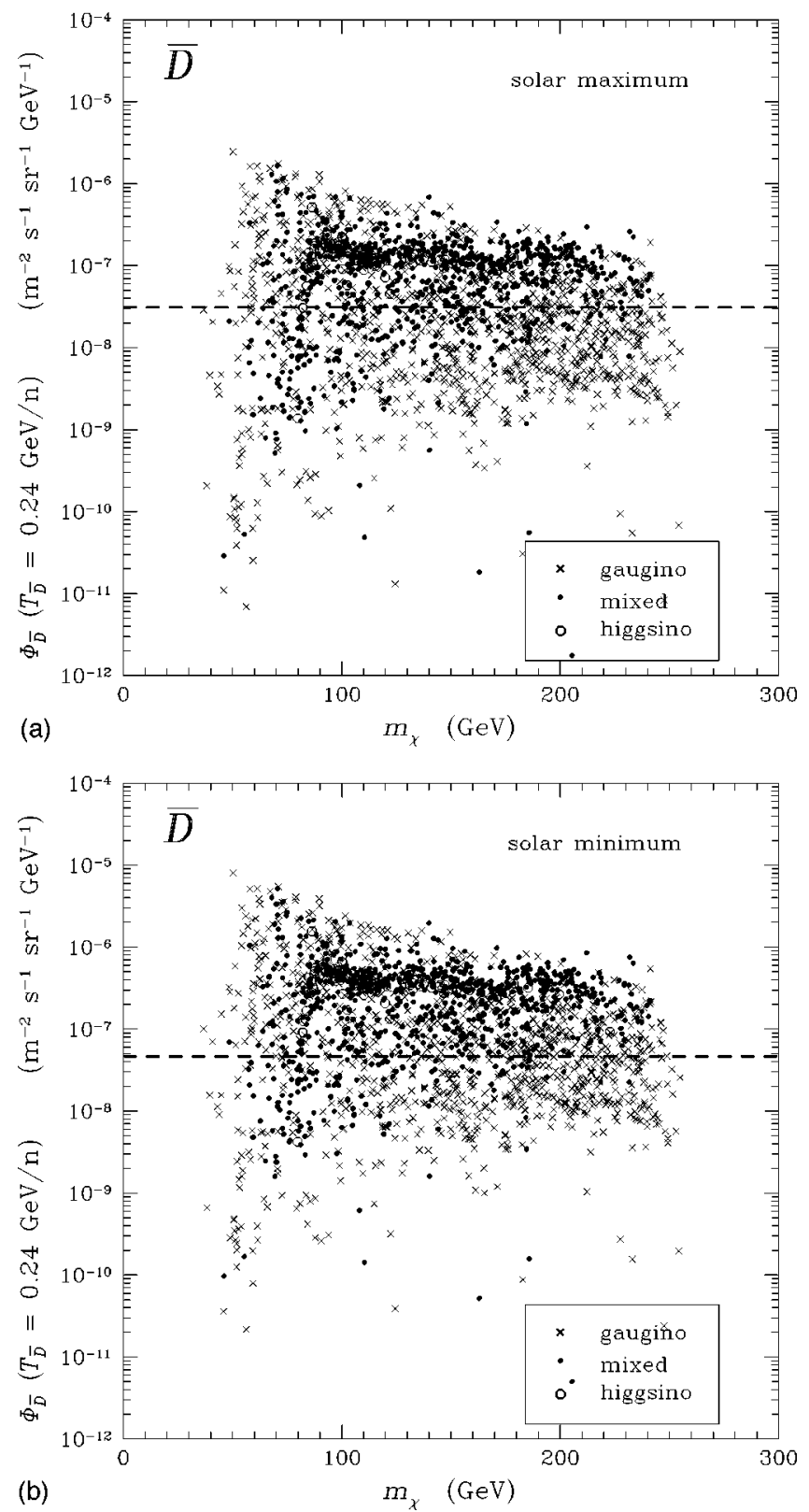

FIG. 7. Scatter plots in the plane $m_{\chi}-\Phi_{\bar{D}}^{\oplus}$. The Earth antideuteron flux $\Phi_{D}^{\oplus}$ has been computed at solar maximum (a) and minimum (b), for a modulated energy of $0.24 \mathrm{GeV} / n$. Configurations lying above the horizontal lines correspond to the detection of at least one antideuteron in the range of interstellar energies $0.1-3$ $\mathrm{GeV}$, by an experiment of the AMS caliber on board ISSA.

In spite of the low fluxes at stake, the antideuteron channel is sensitive to a respectable number of supersymmetric configurations.

Supersymmetric antiprotons are four orders of magnitude more abundant in cosmic rays than antideuterons-see Table I. However, as already discussed, they may be swamped in the background arising from the secondaries. The AMS experiment will collect a large number of antiprotons on board ISSA. Our concern is whether a hypothetical supersymmetric $\bar{p}$ signal may be disentangled from the background. Because the latter still suffers from large theoretical uncertainties, we 


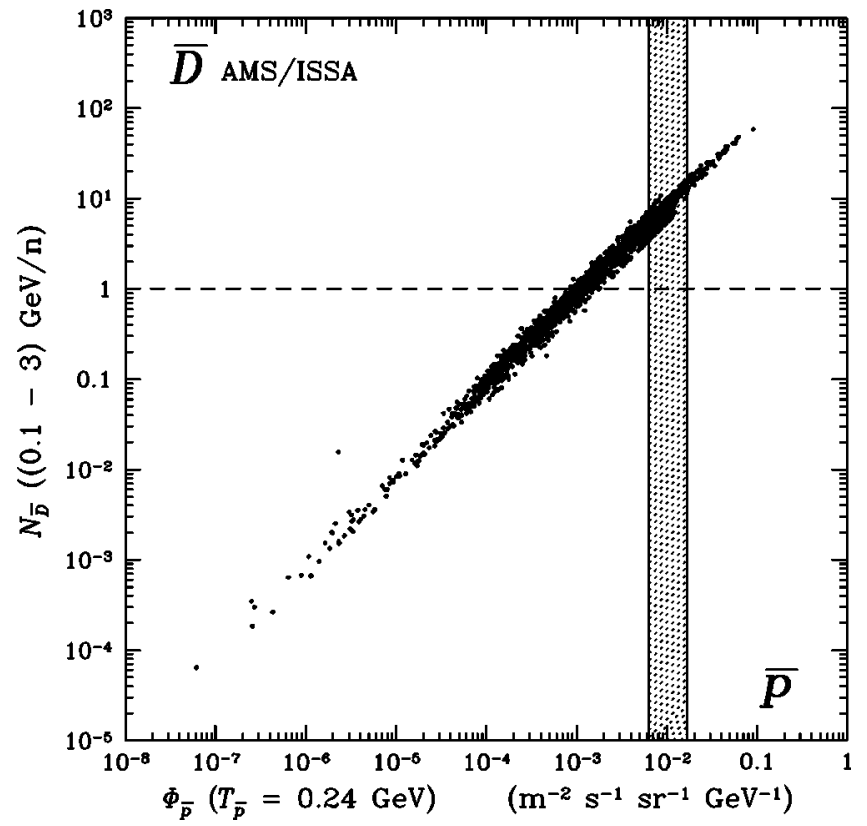

FIG. 8. In this scatter plot, the antideuteron yield $N_{\bar{D}}$ of Fig. 6 is featured against the supersymmetric $\bar{p}$ flux. The antideuteron signal is estimated at solar maximum. This corresponds to the AMS mission on board the space station. The $\bar{p}$ flux is derived on the contrary at solar minimum, in the same conditions as the BESS 95 +97 flights [32] whose combined measurements are indicated by the vertical shaded band for a $\bar{p}$ energy of $0.24 \mathrm{GeV}$. The correlation between the antiproton and antideuteron signals is strong.

are afraid that antiproton searches in cosmic rays are not yet the ultimate probe for the existence of supersymmetric relics in the Milky Way. As discussed in Refs. [4-6], the distribution of secondary antiprotons turns out to be flatter than previously estimated. Therefore, it is still a quite difficult task to ascertain which fraction of the measured antiproton spectrum may be interpreted as a supersymmetric component. Notice however that as soon as the secondary $\bar{p}$ flux is reliably estimated, low-energy antiproton searches will become a more efficient tool. Meanwhile, we must content ourselves with using observations as a mere indication of what a supersymmetric component cannot exceed. The vertical shaded band of Figs. 8 and 9 corresponds actually to the 1- $\sigma$ antiproton flux which the BESS 95 +97 experiments [32] have measured at a $\bar{p}$ kinetic energy of $0.24 \mathrm{GeV}$. In Fig. 8, the supersymmetric antideuteron yield $N_{D}^{-}$has been derived at solar maximum. This corresponds to the conditions of the future AMS mission on board the space station. The antideuteron yield is plotted as a function of the associated supersymmetric $\bar{p}$ flux at Earth. The latter is estimated at solar minimum to conform to the BESS data to which the vertical band refers. The scatter plot of Fig. 8 illustrates the strong correlation between the antideuteron and the antiproton signals, as may be directly guessed from Eq. (15). The horizontal dashed line indicates the level of sensitivity which AMSISSA may reach. Points located above that line but on the left of the shaded vertical band are supersymmetric configurations that are not yet excluded by antiproton searches and for which the antideuteron yield is potentially detectable.

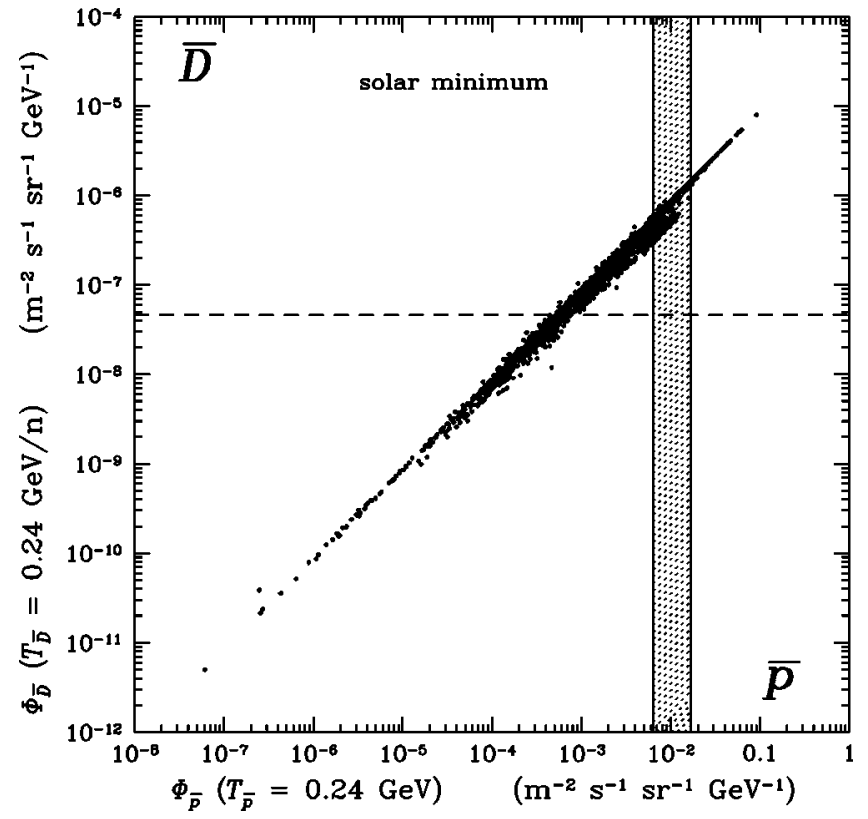

FIG. 9. Both supersymmetric antideuteron and antiproton fluxes at the Earth are plotted against each other. They are modulated at solar minimum, while the energy per nucleon is $T_{D}^{\oplus} / n$ $=0.24 \mathrm{GeV} / n$. As in Fig. 8, the configurations are clearly aligned, hence a strong correlation between the antiproton and antideuteron signals.

The existence of such configurations illustrates the relevance of an antideuteron search at low energies. As shown in Fig. 9, the number of interesting configurations is largest at solar minimum. Both $\bar{D}$ and $\bar{p}$ fluxes at Earth are plotted against each other. Energies have been set equal to $0.24 \mathrm{GeV} / n$. The correlation between the antideuteron and antiproton cosmicray fluxes is once again noticeable.

Once the energy spectrum of the secondary component is no longer spoiled by considerable theoretical uncertainties, measurements of the antiproton cosmic-ray flux will be a powerful way to test the existence of supersymmetric relics in the galactic halo. In the mean time, searches for lowenergy antideuterons appear as a plausible alternative, worth being explored. A dozen spallation antideuterons should be detected by the future AMS experiment on board ISSA above a few $\mathrm{GeV} / n$. For energies less than $\sim 3 \mathrm{GeV} / n$, the $\bar{D}$ spallation component becomes negligible and may be supplanted by a potential supersymmetric signal. We conclude that the discovery of a few low-energy antideuterons should be taken seriously as a clue to the existence of massive neutralinos in the Milky Way. A word of caution, however. Diffusive reacceleration could turn out to be a potential source of flattening for secondary $\bar{D}$ 's. To assess the magnitude of such a flattening requires a complete code which should incorporate a large set of nuclear species, in particular those that are unstable through electron capture. This is beyond the scope of the present analysis which was meant to be exploratory. We nevertheless feel that the case of antideuterons is worth being explored and we hope that our work will motivate further and more refined investigations. 


\section{ACKNOWLEDGMENTS}

We would like to express our gratitude to A. Bottino for stimulating discussions. We also wish to thank R. Battiston and J. P. Vialle for supplying us with useful information pertaining to the AMS experiment. This work was supported by DGICYT under Grant number PB95-1077 and by the TMR network Grant ERBFMRXCT960090 of the European Union.
[1] H. Matsunaga, Ph.D. thesis, University of Tokyo, 1997.

[2] S. Ahlen et al., Nucl. Instrum. Methods Phys. Res. A 350, 351 (1994).

[3] P. Chardonnet, J. Orloff, and P. Salati, Phys. Lett. B 409, 313 (1997).

[4] A. Bottino, F. Donato, N. Fornengo, and P. Salati, Phys. Rev. D 58, 123503 (1998).

[5] L. Bergström, J. Edsjö, and P. Ullio, astro-ph/9902012 (1999).

[6] J. W. Bieber et al., Phys. Rev. Lett. 83, 674 (1999).

[7] L. C. Tan and L. K. Ng, Phys. Rev. D 26, 1179 (1982).

[8] M. A. Braun and V. V. Vechernin, Yad. Fiz. 36, 614 (1982) [Sov. J. Nucl. Phys. 36, 357 (1982)].

[9] W. R. Webber, M. A. Lee, and M. Gupta, Astrophys. J. 390, 96 (1992).

[10] Particle Data Group, C. Caso et al., Eur. Phys. J. C 3, 1 (1998).

[11] A. W. Strong and I. V. Moskalenko, Astrophys. J. 509, 212 (1998).

[12] G. Pelletier (private communication).

[13] V. Ptuskin (private communication).

[14] J. Connell, contribution OG.1.1.10 at the XXVI ICRC conference in Salt Lake City, Utah (1999).

[15] U. Heinbach and M. Simon, Astrophys. J. 441, 209 (1995).

[16] M. Simon and U. Heinbach, Astrophys. J. 456, 519 (1996).

[17] V. S. Berezinskii, S. Bulanov, V. Dogiel, V. Ginzburg, and V. Ptuskin, Astrophysics of Cosmic Rays (North-Holland, Amsterdam, 1990).

[18] P. Chardonnet, G. Mignola, P. Salati, and R. Taillet, Phys. Lett. B 384, 161 (1996).
[19] J. S. Perko, Astron. Astrophys. 184, 119 (1987).

[20] H. P. Nilles, Phys. Rep. 110, 1 (1984); H. E. Haber and G. L. Kane ibid. 117, 75 (1985); R. Barbieri, Riv. Nuovo Cimento 11, 1 (1988).

[21] A. Bottino and N. Fornengo, Lectures given at the Fifth School on Non-Accelerator Particle Astrophysics, ICTP, Trieste, 1998, to appear in the School Proceedings, edited by R. S. Carrigan, Jr., G. Giacomelli, and N. Paver, hep-ph/9904469.

[22] G. Jungman, M. Kamionkowski, and K. Griest, Phys. Rep. 267, 195 (1996).

[23] M. Carena, M. Quirós, and C. E. M. Wagner, Nucl. Phys. B461, 407 (1996).

[24] H. E. Haber, Z. Phys. C 75, 539 (1997).

[25] DELPHI Collaboration, V. Ruhlmann-Kleider, presentation at the LEPC Conference, 1998; L3 Collaboration, R. Clare, ibid.

[26] CLEO Collaboration, S. Glenn, Report No. CLEO CONF 9817, 1998, Proceedings of the International Conference on High Energy Physics, Vancouver, 1998, paper 1011.

[27] ALEPH Collaboration, R. Barate et al., CERN Report No. CERN-EP/98-044, 1998.

[28] A. Bottino, F. Donato, N. Fornengo, and S. Scopel, Phys. Rev. D 59, 095003 (1999).

[29] A. Bottino, C. Favero, N. Fornengo, and G. Mignola, Astropart. Phys. 3, 77 (1995).

[30] L. Bergström and P. Ullio, Nucl. Phys. B504, 27 (1997).

[31] T. Sjöstrand, Comput. Phys. Commun. 39, 347 (1986); 43, 367 (1987); Report No. CERN-TH 6488/92.

[32] H. Matsunaga et al., Phys. Rev. Lett. 81, 4052 (1998); S. Orito, talk given at the 29th International Conference on HighEnergy Physics, Vancouver, 1998. 